\title{
Internet of Things, Internet, Big Data and Airport Services Make Smart Airport Based on $\mathrm{O} 2 \mathrm{O}$ and Humanism
}

\author{
Qi Qi ${ }^{a}$, Zhu Pan ${ }^{\text {b. }}{ }^{*}$ \\ No.10 Xiangyun West Street, Baiyun District, Guangzhou, Guangdong, China \\ aqiqi@caac.net, ${ }^{*}, \mathrm{~b}$ panzhu@caac.net
}

Keywords: Airport services, Smart airport, O2O mode, Internet of things, Service evaluation system.

\begin{abstract}
This paper analyzes the reason why the civil aviation enterprises attach great importance to service quality, but the passengers are not much satisfied. And then it puts forward a scientific solution based on humanism and interconnected intelligent equipment. The paper also defines the concept of a smart airport which is a combination of efficient self-service process with internet of things for offline airport, new information platform for online airport, and new evaluation system with big data. Finally, according to the latest needs of passenger services and the development of software and hardware technology, seven functions of a smart airport are listed, which will completely improve the existing travel experience of civil aviation passengers.
\end{abstract}

\section{Introduction}

Civil aviation industry belongs to the services. China's civil aviation industry is in a period of all-round development with strategic opportunity. Improving the quality of passenger services is an important part of that and also the certain requirement of the whole society. The civil aviation authorities and enterprises like the airlines and airports have always put a high value on that, which is far exceeding any other industry in China. However, in sharp contrast, passengers are not satisfied. The complaints and the conflicts are becoming more and more severe. What is the crux of the matter? It lies in the contradiction between the changed economic environment, the changed passenger needs and the out-of-date management of the civil aviation enterprises. To resolve it, improving the passengers' flight experience will be the key, which is also the trend of China's civil aviation industry development. And to achieve that, the concept of humanism and the application of interconnected intelligent equipment will be the key. This paper will focus on the latter one.

\section{The Practical Significance of a Smart Airport}

Nowadays, the civil aviation transportation suffers from the crowded airports, long queue of waiting passengers, high baggage discrepancy rate, poor service for the flight delays, and competition from high-speed rail, etc. It is urgent to solve these problems efficiently. The overall view of China's civil aviation passenger services will be: on one hand, service difficulty increases because of the growing number of the passengers and increasing level of the individual needs; on the other hand, service capacity decreases because of the lagging process improvement and the lack of internal coordination. The contradiction between the two is irreconcilable. As a service industry, we have no any reason to limit or restrain the reasonable needs of the passengers. What we can and must do is to optimize the coordination between equipment and employees by bettering the work flow. In this way can we expand the passenger services capacity and supply which aims to enhance the consumer experience. In short, the standardized and repetitive work flow should be handed over to the equipment. Otherwise, the personalized and complex task must be dealt with by the employees.

We put forward the concept of smart airport in view of the real need of the passengers during their air travel. Smart airport tends to achieve "man machine integration" by resetting the service process based on internet of things technology, mobile internet technology, big data technology. Since E-ticket has completely changed the traditional mode of civil aviation products marketing in China, 
we have enough reason to expect that smart airport will also bring a disruptive upgrading to the whole industry.

\section{The Model of the Smart Airport}

Humanism and $\mathrm{O} 2 \mathrm{O}$ mode will be the base to build a smart airport.

Humanism is to meet the appeal of both passengers' and civil aviation industry employees'. For the former, it is to provide superior service; for the latter, it is to provide a pleasant and efficient working environment. This is some kind of "win-win" between consumers and providers, and is also a sustainable development way to improve the quality of civil aviation service.

The $\mathrm{O} 2 \mathrm{O}$ mode is to construct an effective combination of an offline airport and an online airport. The offline one is to provide personalized solutions for passengers. For the frequent passengers, we need to rely on the internet of things to realize "easy boarding"-- self-service check-in, paperless and unmanned. And considering the convenience, price and other comprehensive advantages, they will be guided into the "easy boarding" channel. For the less-experienced or first-time passengers(including: children, the elderly, the sick and other special passengers), we need to realize the "enjoyable boarding"-- the paid and exclusive service from check-in to boarding. The online one is to provide all the digital information and inquiring services for passengers' need. It is a full-contact information exchange platform which is timely, personalized, and targeted.

The internet of things, the internet, and big data are the prominent features of a smart airport, and they play their roles in the following fields.

1. Build efficient self-service process with internet of things in offline airport

With the popularity of E-ticket, civil aviation transportation has already implemented self-service in the marketing field. But for the reason of safe operation and business segmentation, the traditional face-to-face mode still maintains in the field of services. From the passengers' arriving at the airport to the flight's departure, the steps like information guide, check-in, baggage check-in, security check, boarding gate registration can certainly be self-serviced as ATM. However, it is hard to realize self-service for the whole process at present due to the isolation of data flow among different links. Based on the internet of things, we can get through the bottleneck of data sharing in all links, and the self-service for the whole process will be the key for the smart airport. Especially for large airports with annual passenger throughput of more than ten million, it is the best way to improve the service capacity and quality.

2. Build new information platform in online airport

In the era of information explosion, people are more and more demanding to control information. The reasons why the satisfying feedback rate of civil aviation service is not high is largely due to the unsatisfied timeliness, accuracy and transparency of information transmission. Considering the safe operation of the whole industry, it is not feasible to open all the related information completely. Therefore, we need to establish an airport terminal based information platform involving the national civil aviation operation system and various of airport services.

3. Build new evaluation system with big data

Service quality improvement aiming at passengers' traveling experience has reached a consensus in the industry. However, there are many problems in specific implementation, rectification and other aspects, which are closely related to the executives judging the feasibility of doing something or changing something in the perspective of the interests of the respective departments. In today's information age, the existing civil aviation service quality evaluation mode which relies on self-examination, superior inspection and rank evaluation is out of date. The right of evaluation has been completely left to the passengers. It is undeniable that the passengers' experience is subjective, but the converged subjective experience must be the real exist. Collect the big data objectively, effectively and systematically, and adhere to problem-oriented method and collaborative innovation. Thus the new civil aviation passenger service evaluation system based on big data will be the foundation for service upgrading and process reengineering. 


\section{The Function Design of the Smart Airport}

Smart airport will be an "man-machine cooperation" system combining software and hardware. Passengers, airports, airlines, service providers and other related participants will involve in it to achieve service providing, information dissemination and interaction. It will make a more refined, personalized and efficient service process. Every passenger will get Airport Mobile Service Terminal(AMST) or download an app and be guided to do what he or she need to do or like to do efficiently in a self-help way.

The smart airport can realize the following seven functions but not be confined to these. And also it will largely better the current situation of civil aviation service.

1.Paperless registration

At the smart airport, when passenger scan his or her 2nd-generation ID card in AMST, the system will identify it and display the self-service check-in interface soon. It allows the passenger to choose seat or just accept a distributed one, and then the electronic boarding pass will be generated. The important flight information like boarding gate number, boarding time and seat number will be displayed on AMST screen. It will save the waiting time in front of the check-in counter and avoid the trouble of losing boarding pass.

2. Personalized navigation

When passenger doesn't know which security check channel is better to choose, AMST will give the exact answer. The real-time queuing monitoring system will distribute the security check channel dynamically and generate the electronic map on AMST screen to guide the passenger. It will effectively help the passenger control his or her time in the security check line.

3. Exclusive passenger mustering

When flight boarding starts, AMST will remind passengers according to the information of the electronic boarding pass, identify the current location of passengers according to the position system, and indicate them the fastest boarding route. In case of special circumstances, passengers can contact the staff in time through information feedback system, and will soon get the best solution from the staff according to the program, which ensures to a largest extent that the flight will not be delayed due to the passengers' delay. It will replace the traditional broadcasting, ensure the flight information to be transmitted to the most needed in the most effective way, while creating a fine sound environment in the waiting hall.

4. Easy-consuming in the departure sterile area

After entering the departure sterile area through security check, passengers may have consumption needs such as catering, shopping, leisure and so on. Effective business promotion information is necessary then. AMST information system can provide that according to the passengers' route to the boarding gate, which meets customers' needs, enriches their waiting experience, and also creates business opportunities for the business suppliers.

5. High efficiency baggage check-in

Passengers can check in the baggage at the self-service counter through AMST baggage handling system. It supports frequent passenger card or bank card to pay for the extra weight. You can also choose to go to the counter for cash payment after check-in. It will maximize the efficiency in the step of baggage check-in.

6. Entire baggage service

The AMST baggage handling system monitors the baggage through the signal source implanted into the baggage card. Before passengers' boarding, it is easy to know whether their checked-in baggage has been shipped or not, which reduces the probability of baggage transportation errors at the departure airport. The system also supports the accurate time checking, location checking, and baggage claiming guiding at the destination airport. It will realize fine management of baggage service and achieve zero error in baggage transportation.

7. Emergency responding service for flight delay

When flight delays, poor information transmission is the main reason for bad service. What caused the delay and how to solve it effectively can not be delivered to the target passengers in time and 
effectively. The AMST emergency responding system provides an effective information transmission channel for flight delay. Dynamic information of delayed flight, ticket changing on line, delay compensation without card, entertainment during waiting, and other functions will greatly improve the passengers' experience during the flight delays.

In addition, AMST will make more and more ideas of civil aviation service innovation into reality, relying on its powerful mobile information technology.

To sum up, the smart airport based on humanism and the concept of $\mathrm{O} 2 \mathrm{O}$ will completely change the existing passenger service process. Self-service mode will improve the passengers' experience and lower the workload of employees at the same time. That's why we call it a win-win revolution that benefits the passengers and the employees. The most successful service is active, positive and from the bottom of heart. In fact, China's civil aviation passenger services have been in innovation. The smart airport is not far away from us.

\section{Acknowledgements}

This research was financially supported by Safety Capacity Construction Projects of Civil Aviation Administration of China, 2017.

\section{References}

[1]. Qi Qi, Linli Yao, Evaluation method of civil aviation service quality based on passenger experience identification technology, Civil Aviation Management, 323 (2017) 18-22.

[2]. Qi Qi, Linli Yao, Innovation of evaluation of civil aviation service quality based on passenger experience identification technology, China Civil Aviation, 253 (2017) 73-74.

[3]. Qi Qi, Guangping Ji, Improve the quality of baggage service with the internet of things, China Civil Aviation Newspaper, 2016-10-14(2).

[4]. Qi Qi, Gongbo Chai, The essence of Southwest Airlines model — coordination, China Civil Aviation, 191 (2014) 73-74.

[5]. Fang Yang, Qi Qi, The Check-in and Baggage Transport (second edition), National Defend Industry Press, Beijing, 2015.

[6]. Qi Qi, The Outlook of Industry Standard — the Trend of China's Civil Aviation Development, National Defend Industry Press, Beijing, 2016.

[7]. Wujun Liu, Terminal Building Planning, Shanghai Scientific \& Technical Publishers, Shanghai, 2017.

[8]. Yanhua Li, Economic Theory and Practice of Air Transport, China Civil Aviation Press, Beijing, 2017.

[9]. Information on http://www.caac.gov.cn

[10]. Information on http://www.carnoc.com.cn 